\title{
Highly Stable Hysteresis-Free Carbon Nanotube Thin-Film Transistors by Fluorocarbon Polymer Encapsulation
}

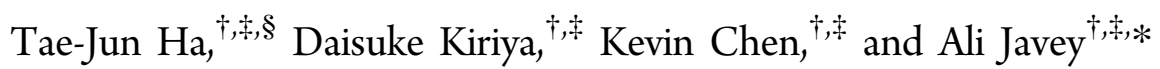 \\ ${ }^{\dagger}$ Electrical Engineering and Computer Sciences, University of California, Berkeley, California 94720, United States \\ ${ }^{\ddagger}$ Materials Sciences Division, Lawrence Berkeley National Laboratory, Berkeley, California 94720, United States
}

ABSTRACT: We report hysteresis-free carbon nanotube thin-film transistors (CNT-TFTs) employing a fluorocarbon polymer (Teflon-AF) as an encapsulation layer. Such fluorocarbon encapsulation improves device uniformity with excellent operation stability in air and even in water. The fluoropolymers possess high hydrophobicity for efficient removal of water molecules from the vicinity of nanotubes, which are known to induce charge trapping. In addition, the strong dipole associated with the carbon-fluorine bonds can provide effective screening of the charge carriers in nanotubes
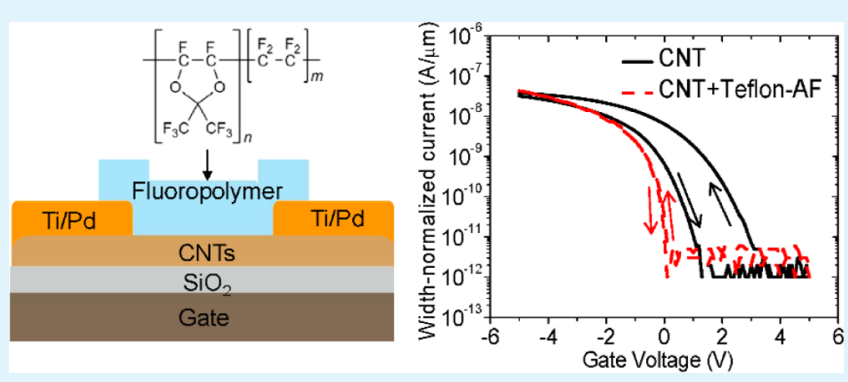
from various trap states in the substrate. We also report on the extended applications of encapsulation with Teflon-AF for the realization of dual-gate CNT-TFTs, where one gate is used to control the threshold voltage and the other to switch the device. The fluorocarbon encapsulation technique presents a promising approach for enhanced device reliability, which is critical for future system-level electronics based on CNTs.

KEYWORDS: carbon nanotubes, electronics, stability, hydrophobic, passivation

\section{INTRODUCTION}

Given their superb electrical, mechanical, and chemical properties, single-walled carbon nanotubes (CNTs) have been extensively explored for a number of electronic applications. $^{1-12}$ In particular, solution processing of random networks of semiconductor-enriched nanotubes for thin-film transistors (TFTs) has been shown to be highly promising for large-area and/or printed electronics. ${ }^{13-22}$ CNT-TFTs exhibit high mobilities, well surpassing those of other TFT channel materials such as amorphous silicon and organic semiconductors. As a result, high-current drives at low voltages are obtained, making them suitable for low-power logic circuits on plastics and active-matrix backplanes for a wide range of systems, including flexible displays, imagers, and interactive sensor networks. ${ }^{23-28}$ However, instability of device characteristics caused by environmental effects presents a challenge toward further advancement of the field. ${ }^{29-32}$

For any device technology, developing reliable encapsulation layers is essential for removing interactions with molecules in the environment, thereby providing stable operation over time. ${ }^{33-36}$ This is particularly important for material systems with molecular-scale dimensions, such as single-walled carbon nanotubes where all of the atoms are on the surface, hence making them highly susceptible to the environment. ${ }^{37,38}$ Specifically, large hysteresis has been commonly observed in the current versus gate voltage characteristics of nanotube devices. Hyteresis is highly undesirable for reliable transistor operation and logic circuit design as it leads to a shift in the threshold voltage, $V_{\mathrm{th}}$ as a function of the voltage sweeping direction and range. Hysteresis in a transistor can arise from multiple factors, including (i) trap states in the dielectric or semiconductor/dielectric interface ${ }^{39}$ and (ii) trap charges induced by water molecules absorbed onto or in the vincitiy of the semiconductor channel. ${ }^{30-32,40-43}$ A previous study of transistors based on pristine single nanotubes grown by chemical vapor deposition (CVD) on clean $\mathrm{Si} / \mathrm{SiO}_{2}$ substrates has shown that the observed hysteresis is due to charge trapping by water molecules near the nantoubes. ${ }^{31,44}$ For such devices, poly(methyl methacrylate) (PMMA) passivation was shown to be effective in removing water molecules and hence the hysteresis effect. ${ }^{31,43}$ However, the use of the same encapsulation technique for TFTs based on solution-processed CNT networks fails to remove the device hysteresis as shown later in this work. For such devices, the surface of the substrate is functionalized with amine groups for nanotube adhesion from the solution suspension. ${ }^{45,46}$ Amine functionalization leads to a more hydrophilic surface as compared to pristine $\mathrm{SiO}_{2}$, thereby facilitating stronger absorption and binding of water molecules on the surface. As a result, efficient removal of water molecules is challenging. Recent reports have also shown that certain oxide dielectrics deposited on nanotubes can reduce hysteresis by removing water molecules from the surface. $^{38}$ Others have proposed functionalizing the surface onto which the CNTs are deposited to prevent adsorption of water $^{47}$ and trapped charges. ${ }^{48,49}$ However, to date, minimal attention has been given to the reliability of these techniques in

Received: March 5, 2014

Accepted: May 5, 2014

Published: May 5, 2014 
terms of device to device variation and stability over time upon exposure to ambient air or water. Given the rise of solutionprocessed CNT-TFTs as a promising technology platform for large-area sensor and electronic applications, there is a critical need to develop a simple, reliable encapsulation technology for these devices to enhance uniformity and stability.

In this work, we demonstrate hysteresis-free and highly robust CNT-TFTs by encapsulation with solution-processed fluoropolymers. Owing to their high hydrophobicity, fluoropolymers effectively repel water molecules from the surface of the substrate. Furthermore, due to the low permeability of fluoropolymers to water molecules and other molecular species, the devices exhibit excellent long-term stability in ambient environment and can maintain their electrical characteristics in a high-humidity environment or even under water. In addition, we show the benefit of fluorocarbon passivation on improving the performance uniformity of the devices.

\section{EXPERIMENTAL SECTION}

The device schematic of a CNT-TFT encapsulated with a fluoropolymer Teflon-AF (poly[4,5-difluoro-2,2-bis(trifluoromethyl)1,3-dioxole-co-tetrafluoroethylene]; Dupont Co. 400S2-100-1, 1\% solution) and its corresponding chemical structure are shown in Figure 1a,b, respectively. Teflon-AF exhibits excellent hydrophobicity
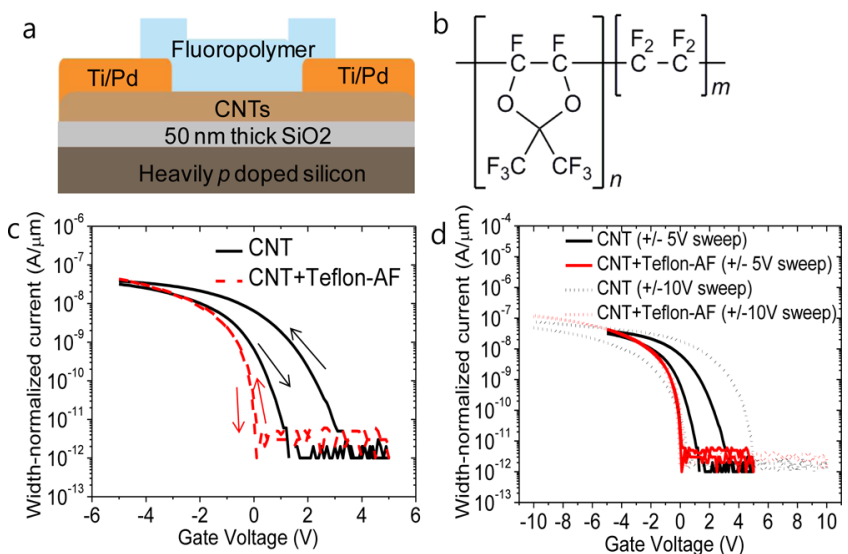

Figure 1. (a) Device schematic of a CNT-TFT encapsulated with a fluoropolymer Teflon-AF and (b) its corresponding chemical structure ( $m$ and $n$ represent integers). (c) Transfer curves of representative CNT-TFTs with and without encapsulation by Teflon-AF for a voltage scan range of $\pm 5 \mathrm{~V}$. (d) Hysteresis characteristics of CNT-TFTs with different gate voltage sweep ranges $( \pm 5$ and $\pm 10 \mathrm{~V})$ with and without Teflon-AF encapsulation.

with a water contact angle of $105^{\circ}$, water absorption of $<0.01 \%$, and low gas permeability for most species, including water molecules. ${ }^{50}$ Our device fabrication starts with a $50 \mathrm{~nm}$ thick thermally grown $\mathrm{SiO}_{2}$ on a heavily doped p-type $\mathrm{Si}$ wafer which acts as the gate dielectric and gate electrode, respectively. Wafers were rinsed with acetone, methanol, and isopropyl alcohol, dried with nitrogen gas flow, and baked in an oven at $120{ }^{\circ} \mathrm{C}$ for $10 \mathrm{~min}$ in order to remove organic contaminants from the surface. The samples were then treated with oxygen plasma at $120 \mathrm{~W}$ for $2 \mathrm{~min}$ followed by drop-casting of poly-Llysine (Sigma-Aldrich; $0.1 \%\left(\mathrm{w} / \mathrm{v}\right.$ ) in $\mathrm{H}_{2} \mathrm{O}$ ) solution onto the surface of the $\mathrm{SiO}_{2}$ for $5 \mathrm{~min}$, and a rinse with deionized (DI) water. Following this surface functionalization, an as-supplied $99 \%$ semiconducting single-walled CNT suspension (NanoIntegris Inc.) was drop-casted for $60 \mathrm{~min}$ followed by a rinse with DI water. This process leads to a dense and uniform assembly of nanotube random networks on the $\mathrm{SiO}_{2}$ surface as we previously reported. ${ }^{24-26}$ A $0.5 \mathrm{~nm}$ thick titanium ( $\mathrm{Ti}) / 40 \mathrm{~nm}$ thick palladium (Pd) bilayer as source/drain electrodes was deposited by using a shadow mask and electron-beam evaporation under a vacuum of $\sim 10^{-6}$ Torr. Finally, a $100 \mathrm{~nm}$ thick Teflon-AF encapsulation layer was formed on the surface by spincoating ( $2500 \mathrm{rpm}$ for $1 \mathrm{~min}$ ) and thermally annealed in an oven by gradually increasing the temperature from 30 to $200{ }^{\circ} \mathrm{C}$ over a span of $1 \mathrm{~h}$ in a nitrogen atmosphere. The annealing was performed to remove all of the solvents as well as water molecules on the surface of the substrate, enhance the adherence of the polymer on the surface of the substrate, and provide a smoother surface coverage. CNT-TFTs fabricated for this study possess channel widths and lengths of 1000 and $50 \mu \mathrm{m}$, respectively. Electrical characteristics for all samples were measured in ambient air unless otherwise noted. The applied voltage between drain and source electrodes was $-5 \mathrm{~V}$.

\section{RESULTS AND DISCUSSION}

Figure 1c shows the transfer curves of representative CNTTFTs with and without encapsulation by Teflon-AF. The measurements were performed by sweeping the gate voltage between -5 and $+5 \mathrm{~V}$ at a scan rate of $0.5 \mathrm{~V} / \mathrm{s}$ in the forward and backward directions as depicted by the arrows in the plot. The device without encapsulation clearly depicts a large hysteresis, with a threshold voltage difference of $\sim 1.6 \mathrm{~V}$ between the two scanning directions. This undesired hysteresis is a characteristic that is often observed in nanotube transistors. On the other hand, upon encapsulation with Teflon-AF, the threshold voltage hysteresis is drastically reduced to $<0.1 \mathrm{~V}$ for the same voltage scan rate and range. Owing to its excellent chemical properties, previous studies have shown that fluoropolymers can minimize the voltage hysteresis for organic transistors. ${ }^{51,52}$ Hysteresis is affected by the magnitude of the gate bias sweep because the number of initially trapped charge carriers depends on the gate bias. Figure $1 \mathrm{~d}$ shows the hysteresis characteristics for gate voltage scans of \pm 5 and \pm 10 $\mathrm{V}$, with and without Teflon-AF encapsulation. Without encapsulation, the hysteresis window increases from 1.6 to $4.5 \mathrm{~V}$ as the scan range is increased from \pm 5 to $\pm 10 \mathrm{~V}$. Here, the hysteresis window is defined by the voltage difference between the forward and the reverse sweep at the drain current of $10^{-7} \mathrm{~A}$. However, upon encapsulation, the device exhibits almost hysteresis-free characteristics with a hysteresis window of less than $0.1 \mathrm{~V}$ and a constant threshold voltage, regardless of the magnitude of the applied gate bias range.

In addition to the elimination of hysteresis, the $V_{\text {th }}$ of the encapsulated device is shifted toward the negative gate voltage, resulting in the device being fully OFF at $V_{\mathrm{gs}}=0 \mathrm{~V}$. This enhancement mode operation is highly desired for the use of CNT-TFTs for active-matrix backplanes and digital logics. In contrast, for the nanotube processing conditions used here, the device without encapsulation shows a slightly positive gate voltage, corresponding to depletion mode operation where the device is still partially $\mathrm{ON}$ at $V_{\mathrm{gs}}=0 \mathrm{~V}$. This can be attributed to the removal of various absorbed ambient molecules on the surface of nanotubes by Teflon-AF that can induce surface charge transfer which shift the threshold voltage in the positive direction. Additionally, after encapsulation with Teflon-AF, the subthreshold swing, SS, decreases from $0.61 \mathrm{~V} /$ decade to 0.13 $\mathrm{V} /$ decade compared to that of the original CNT-TFT without encapsulation.

The improved SS by encapsulation can be attributed to the suppression of the interfacial interaction between charge carriers in the nanotube channel and trap charges related to water molecules. The effective trap density of states $\left(N_{\text {trap density }}\right)$ before and after encapsulation can be estimated from the SS analysis using the following expression ${ }^{53}$ 


$$
\mathrm{SS}=\frac{k T \ln 10}{e}\left[1+\frac{e^{2}}{C_{\mathrm{ox}}} N_{\text {trap density }}\right]
$$

where $k$ is the Boltzmann constant, $T$ is the temperature, and $C_{\text {ox }}$ is a capacitance per unit area of gate dielectric calculated by using the analytical equation as follows: ${ }^{3,54}$

$$
C_{\mathrm{ox}}=\left\{C_{\mathrm{Q}}^{-1}+\frac{1}{2 \pi \varepsilon_{\mathrm{o}} \varepsilon_{\mathrm{ox}}} \ln \left[\frac{2 \Lambda_{\mathrm{o}} \sinh \left(\frac{2 \pi t_{\mathrm{ox}}}{\Lambda_{\mathrm{o}}}\right)}{\pi d}\right]\right\}^{-1} \Lambda_{\mathrm{o}}^{-1}
$$

where $1 / \Lambda_{\mathrm{o}}$ is the density of nanotubes which is counted from atomic force microscopy, $C_{\mathrm{Q}}=4.0 \times 10^{-10} \mathrm{~F} / \mathrm{m}$ is the quantum capacitance of nanotubes, $\varepsilon_{\mathrm{o}}$ is the relative permittivity, $\varepsilon_{\mathrm{ox}}=$ 3.9 is a dielectric constant of $\mathrm{SiO}_{2}, t_{\text {ox }}$ is the effective oxide thickness, and $d$ is the diameter of the nanotubes, which range from 1 to $1.4 \mathrm{~nm}$. The calculated capacitance value is $3.1 \times$ $10^{-8} \mathrm{~F} / \mathrm{m}$. After encapsulation with Teflon-AF, the effective trap density decreases from $\sim 1.8 \times 10^{12}$ to $2.2 \times 10^{11} \mathrm{~cm}^{-2}$ $\mathrm{eV}^{-1}$. In addition to water removal, a possible explanation for the origin of such improvement in CNT-TFTs encapsulated with Teflon-AF can be the strong dipole-dipole interaction of the carbon-fluorine bonds which serve to screen out the effect of interfacial interaction between charge carriers and various interface trap states, including those associated with the hydroxyl and amine groups of the $\mathrm{SiO}_{2}$ substrate. ${ }^{55,56}$

Optimization of the encapsulation process is critical. In this regard, we explored the effect of Teflon-AF layer thickness as well as its thermal annealing temperature. Figure $2 \mathrm{a}$ shows the
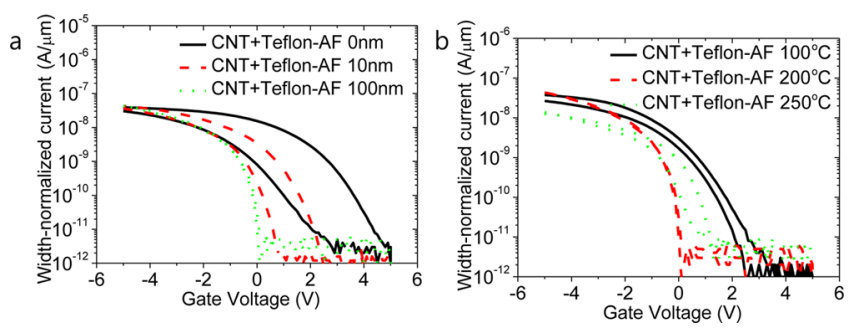

Figure 2. (a) Double-sweep transfer characteristics for a CNT-TFT without encapsulation and with $10 \mathrm{~nm}$ and $100 \mathrm{~nm}$ thick Teflon-AF. (b) Transfer characteristics as a function of Teflon-AF annealing temperature.

double-sweep transfer characteristics of a CNT-TFT without encapsulation and with $10 \mathrm{~nm}$ and $100 \mathrm{~nm}$ thick Teflon-AF. The hysteresis is drastically reduced as the thickness of the Teflon-AF is increased with the hysteresis being nearly diminished for a $100 \mathrm{~nm}$ thick layer. This represents the minimal thickness that is needed to provide a pinhole-free film with conformal coverage to effectively shield the nanotube active channel from outside environmental effects. ${ }^{57}$ In addition, the effect of the annealing temperature of the Teflon-AF was explored. We examined different annealing temperatures ranging from 100 to $250{ }^{\circ} \mathrm{C}$, as shown in Figure $2 \mathrm{~b}$. From the transfer curves it can be seen that the sample annealed at $200{ }^{\circ} \mathrm{C}$ exhibits hysteresis-free behavior while those annealed at lower or higher temperatures exhibit larger hysteresis, but still smaller than samples without encapsulation. When the annealing temperature is below $100{ }^{\circ} \mathrm{C}$, the solvent residues are not completely removed and the coverage and adherence of the polymer on the surface are not optimal. On the other hand, when the temperature is too high (e.g., $\sim 250$ ${ }^{\circ} \mathrm{C}$ ), we speculate that incorporation of nanotube surfactant residues and/or amine surface functional groups into the Teflon-AF may result in degradation of performance. From this result, it can be seen that thermal treatment at $200{ }^{\circ} \mathrm{C}$ is optimal for Teflon-AF encapsulation of CNT-TFTs.

Figure 3 shows the statistic plots of the (a) threshold voltage, (b) hysteresis window, (c) SS, and (d) mobility for 42 different
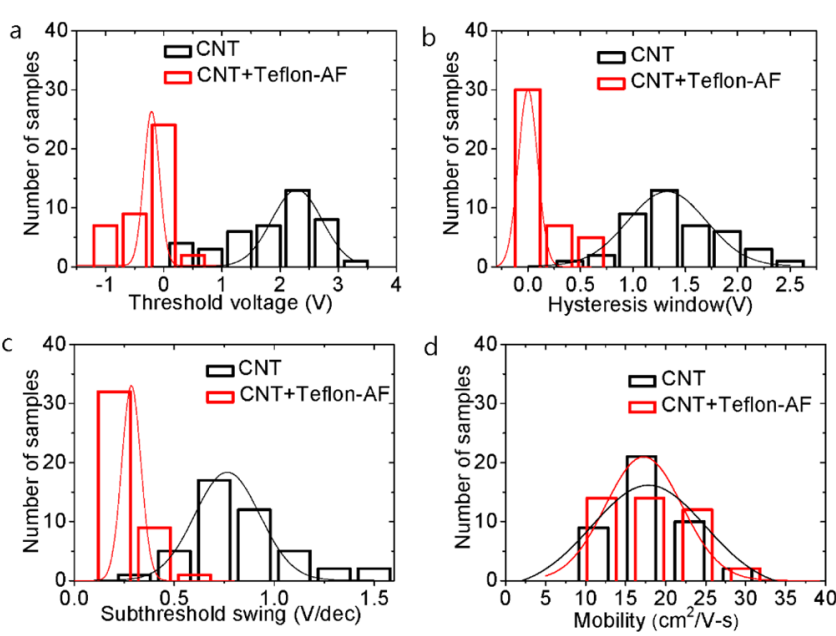

Figure 3. Histogram plots of (a) threshold voltage, (b) hysteresis window, (c) subthreshold swing, and (d) mobility for 42 different TFTs fabricated in different batches at different times with and without Teflon-AF encapsulation.

TFTs fabricated in different batches at different times. The fluorocarbon encapsulation conclusively reduces the hysteresis from $1.32 \pm 0.37$ to $0 \pm 0.09 \mathrm{~V}$ for a $V_{\mathrm{g}}$ scan range of $\pm 5 \mathrm{~V}$ and shifts the $V_{\text {th }}$ from $2.28 \mathrm{~V} \pm 0.43$ to $-0.2 \pm 0.13 \mathrm{~V}$. In addition, SS is improved from $0.76 \pm 0.16$ to $0.28 \pm 0.04 \mathrm{~V} /$ decade whereas the mobility is maintained from $17.85 \pm 7.12$ to 17.26 $\pm 4.77 \mathrm{~cm}^{2} /(\mathrm{V} \cdot \mathrm{s})$. Importantly, the data depict the improved uniformity of transistors upon Teflon-AF encapsulation, especially in terms of $V_{\mathrm{th}}$, mobility, and SS, which is critical for practical use of CNT-TFTs.

Figure 4a shows the electrical characteristics of an encapsulated TFT after long exposure time to ambient air.

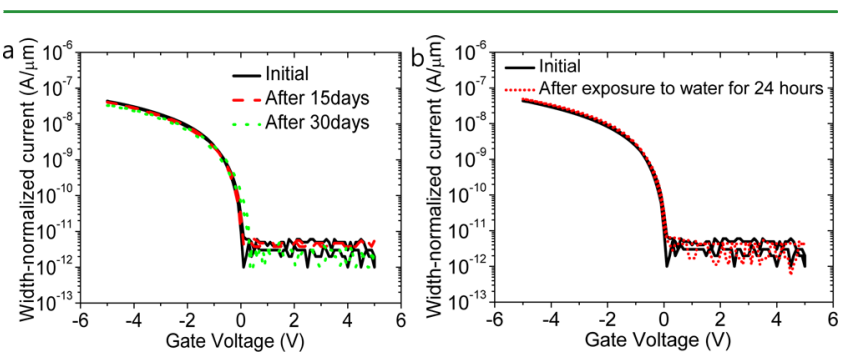

Figure 4. Electrical characteristics of an encapsulated TFT (a) after 15 and 30 days exposure to ambient air and (b) after immersion in water for $24 \mathrm{~h}$ demonstrating the excellent operation stability.

The transfer characteristic remains nearly unchanged with no hysteresis and a constant $V_{\mathrm{th}}$, even after exposure of the device to ambient air for 30 days. Such long-term stability in CNTTFTs is a critical merit for passivation and/or packaging for practical applications. Furthermore, the devices are waterproof. After immersion of the encapsulated device under water for 24 
$h$, the device characteristics remain unchanged without hysteresis, as shown in Figure $4 \mathrm{~b}$. This result highlights the low permittivity of Teflon-AF to water molecules, again highlighting its effectiveness as an encapsulation layer for CNT-TFTs.

Next, we explored other polymer encapsulation layers for CNT-TFTs. In addition to the Teflon-AF discussed above, we explored a $90 \mathrm{~nm}$ thick fluoropolymer CYTOP, $400 \mathrm{~nm}$ thick PMMA, and $500 \mathrm{~nm}$ thick parylene-C, as shown in Figure 5a.

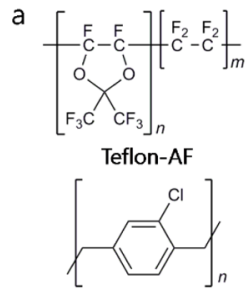

Parylene-C

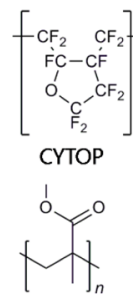

PMMA

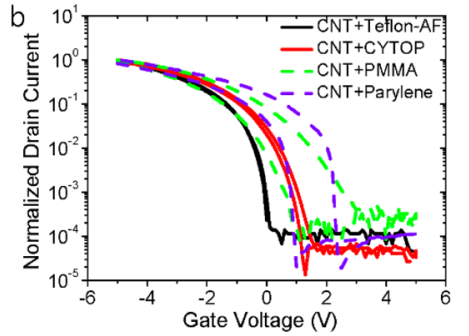

Figure 5. (a) Chemical structure of encapsulants Teflon-AF, CYTOP, PMMA, and parylene-C explored in this study ( $m$ and $n$ represent integers). (b) Double-sweep transfer characteristics for CNT-TFTs employing different encapsulants. The results show only fluoropolymers are effective encapsulants in removing the hysteresis.

The first two polymers were deposited by spin-coating and thermally annealed in vacuum at $200{ }^{\circ} \mathrm{C}$ while parylene- $\mathrm{C}$ was deposited at room temperature using a vapor-phase transport process. As can be seen from Figure $5 b$, the fluoropolymer CYTOP is also a promising encapsulant with the passivated devices exhibiting minimal hysteresis, although slightly larger than that measured from the Teflon-AF devices. On the other hand, both PMMA and parylene-C encapsulation are ineffective for complete removal of hysteresis in the CNT-TFTs. This is in contrast to the results obtained from single-nanotube transistors that were grown by CVD directly on the substrate. We speculate that the existence of surface functionalization on the surface of the substrate (e.g., amine groups) ${ }^{45,46}$ cause strong binding of water molecules onto the surface which do not get readily removed or repelled by PMMA and parylene-C. Instead, highly hydrophobic fluoropolymers, such as Teflon-AF and CYTOP are more effective in removing the various environmental effects.

It should be noted that polymer residues, such as photoresist contaminants on the surface of nanotubes can cause additional hysteresis. ${ }^{35-43}$ In this regard, if photoresist is used for nanotube processing, careful attention needs to be paid to the surface cleaning steps to ensure that the surface is as clean as possible. In this work, we focused on the use of shadow mask for the metal contact patterning of nanotube devices, without the need to use photolithography. Therefore, the nanotubes are not exposed to photoresist during processing.

Finally, we extended the use of the Teflon-AF encapsulation layer as a top-gate dielectric for dual-gate operation by fabricating a patterned gate electrode on the top surface. Figure 6 shows that the transfer characteristics of the CNTTFTs measured by sweeping the back-gate voltage while stepping the top-gate voltage in $2 \mathrm{~V}$ increments. It can be seen that the top-gate voltage modulates the threshold voltage of the device. This resembles the operation of Si-based dual-gate transistors, ${ }^{58}$ where one gate is used for switching operation of the device (in this case, the back-gate) while the second gate (i.e., top-gate) is used for modulating the threshold voltage.

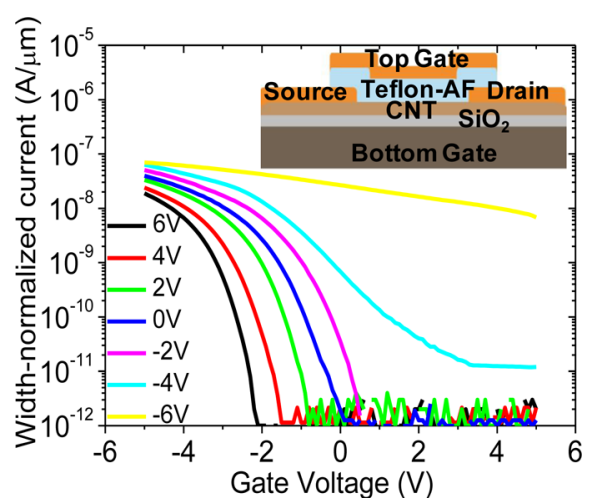

Figure 6. Transfer characteristics of CNT-TFTs configured into a double-gate structure measured by sweeping the back-gate voltage while stepping the top-gate voltage in $2 \mathrm{~V}$ increments.

Using this dual-gate structure, we demonstrate that we can obtain both depletion and enhancement mode device operations by simply applying the proper top-gate voltage. Specifically, the CNT-TFTs are operating in the depletion mode for a top-gate voltage of $<-2 \mathrm{~V}$ and in the enhancement mode for $>0 \mathrm{~V}$. This provides more flexibility in designing complex integrated circuits based on CNT-FETs in the future.

\section{CONCLUSION}

In summary, we have demonstrated hysteresis-free CNT-TFTs employing a solution-processed fluorocarbon polymer, Teflon$\mathrm{AF}$, as an encapsulation layer. By optimizing fabrication conditions, we observed improvement in the key device metrics including the uniformity and stability, even when the devices are immersed under water. The effective encapsulation by Teflon-AF is attributed to the removal of water from the vicinity of the nanotubes while screening the various trap states through its strong dipole-dipole carbon-fluorine bonds. The work presents an important advance toward further control of the device properties of CNT-TFTs with excellent operation stability through proper encapsulation and can enable exploration of more sophisticated electronic systems in the future.

\section{AUTHOR INFORMATION}

\section{Corresponding Author}

*E-mail: ajavey@eecs.berkeley.edu.

\section{Present Address}

${ }^{\S}$ Department of Electronic Materials Engineering, Kwangwoon University, Seoul 139-701, Republic of Korea.

\section{Notes}

The authors declare no competing financial interest.

\section{ACKNOWLEDGMENTS}

This work was supported by the NSF NASCENT Center.

\section{REFERENCES}

(1) Tans, S. J.; Verschueren, A. R. M.; Dekker, C. RoomTemperature Transistor Based on a Single Carbon Nanotube. Nature 1998, 393, 49-52.

(2) Javey, A.; Guo, J.; Wang, Q.; Lundstrom, M.; Dai, H. Ballistic Carbon Nanotube Transistors. Nature 2003, 424, 654-657.

(3) Kang, S. J.; Kocabas, C.; Ozel, T.; Shim, M.; Pimparkar, N.; Alam, M. A.; Rotkin, S. V.; Rogers, J. A. High-Performance Electronics Using 
Dense, Perfectly Aligned Arrarys of Single-Walled Carbon Nanotubes. Nat. Nanotechnol. 2007, 2, 230-236.

(4) Baughman, R. H.; Zakhidov, A. A.; de Heer, W. A. Carbon Nanotubes-The Route Toward Applications. Science 2002, 297, 787-792.

(5) Engel, M.; Small, J. P.; Steiner, M.; Freitag, M.; Green, A. A.; Hersam, M. C.; Avouris, P. Thin Film Nanotube Transistors Based on Self-Assembled, Aligned, Semiconducting Carbon Nanotube Arrays. ACS Nano 2008, 2, 2445-2452.

(6) Hersam, M. C. Progress Towards Monodisperse Single-Walled Carbon Nanotubes. Nat. Nanotechnol. 2008, 3, 387-394.

(7) Iijima, S.; Brabec, C.; Maiti, A.; Bernholc, J. Structural Flexibility of Carbon Nanotubes. J. Chem. Phys. 1996, 104, 2089-2092.

(8) Artukovic, E.; Kaempgen, M.; Hecht, D. S.; Roth, S.; Gruner, G. Transparent and Flexible Carbon Nanotube Transistors. Nano Lett. 2005, 5, 757-760.

(9) Bekyarova, E.; Itkis, M. E.; Cabrera, N.; Zhao, B.; Yu, A.; Gao, J.; Haddon, R. C. Electronic Properties of Single-Walled Carbon Nanotube Networks. J. Am. Chem. Soc. 2005, 127, 5990-5995.

(10) Odom, T. W.; Huang, J.-L.; Kim, P.; Lieber, C. M. Atomic Structure and Electronic Properties of Single-Walled Carbon Nanotubes. Nature 1997, 391, 62-64.

(11) Zhang, J.; Gui, H.; Liu, B.; Liu, J.; Zhou, C. Comparative Study of Gel-Based Separated Arcdischarge, HiPCO, and CoMoCAT Carbon Nanotubes for Macroelectronic Applications. Nano Res. 2013, 6, 906-920.

(12) Wang, C.; Zhang, J.; Zhou, C. Macroelectronic Integrated Circuits Using High-Performance Separated Carbon Nanotube ThinFilm Transistors. ACS Nano 2010, 4, 7123-7132.

(13) Ha, M.; Seo, J.-W. T.; Prabhumirashi, P. L.; Zhang, W.; Geier, M. L.; Renn, M. J.; Kim, C. H.; Hersam, M. C.; Frisbie, C. D. Aerosol Jet Printed, Low Voltage, Electrolyte Gated Carbon Nanotube Ring Oscillators with Sub-5 $\mu$ s Stage Delays. Nano Lett. 2013, 13, 954-960.

(14) Chen, P.; Fu, Y.; Aminirad, R.; Wang, C.; Zhang, J.; Wang, K.; Galatsis, K.; Zhou, C. Fully Printed Separated Carbon Nanotube Thin Film Transistor Circuits and Its Application in Organic Light Emitting Diode Control. Nano Lett. 2011, 11, 5301-5308.

(15) Ha, M.; Xia, Y.; Green, A. A.; Zhang, W.; Renn, M. J.; Kim, C. H.; Hersam, M. C.; Frisbie, C. D. Printed, Sub-3V Digital Circuits on Plastic from Aqueous Carbon Nanotube Inks. ACS Nano 2010, 4, $4388-4395$.

(16) Zhang, J.; Fu, Y.; Wang, C.; Chen, P.-C.; Liu, Z.; Wei, W.; Wu, C.; Thompson, M. E.; Zhou, C. Separated Carbon Nanotube Macroelectronics for Active Matrix Organic Light-Emitting Diode Displays. Nano Lett. 2011, 11, 4852-4858.

(17) Wang, C.; Takei, K.; Takahashi, T.; Javey, A. Carbon Nanotube Electronics-Moving Forward. Chem. Soc. Rev. 2013, 42, 2592.

(18) Bradley, K.; Gabriel, J.-C. P.; Gruner, G. Flexible nanotube electronics. Nano Lett. 2003, 3, 1353-1355.

(19) Wang, C.; Zhang, J.; Ryu, K.; Badmaev, A.; De Arco, L. G.; Zhou, C. Wafer-Scale Fabrication of Separated Carbon Nanotube Thin-Film Transistors for Display Applications. Nano Lett. 2009, 9 (12), 4285-4291.

(20) Behnam, A.; Sangwan, V. K.; Zhong, X.; Lian, F.; Estrada, D.; Jariwala, D.; Hoag, A. J.; Lauhon, L. J.; Marks, T. J.; Hersam, M. C.; Pop, E. High-Field Transport and Thermal Reliability of Sorted Carbon Nanotube Network Devices. ACS Nano 2013, 7 (1), 482-490.

(21) Rouhi, N.; Jain, D.; Burke, P. J. High-Performance Semiconducting Nanotube Inks: Progress and Prospects. ACS Nano 2011, 5, 8471-8487.

(22) Snow, E. S.; Novak, J. P.; Campbell, P. M.; Park, D. Random. Networks of Carbon Nanotubes as an Electronic Material. Appl. Phys. Lett. 2003, 82, 2145-2147.

(23) Zhang, D.; Ryu, K.; Liu, X.; Polikarpov, E.; Ly, J.; Tompson, M. E.; Zhou, C. Transparent, Conductive, and Flexible Carbon Nanotube Films and Their Application in Organic Light-Emitting Diodes. Nano Lett. 2006, 6, 1880-1886.

(24) Takahashi, T.; Yu, Z.; Chen, K.; Kiriya, D.; Wang, C.; Takei, K.; Shiraki, H.; Chen, T.; Ma, B.; Javey, A. Carbon Nanotube Active-
Matrix Backplanes for Mechanically Flexible Visible Light and X-ray Imagers. Nano Lett. 2013, 13, 5425-5430.

(25) Lau, P. H.; Takei, K.; Wang, C.; Ju, Y.; Kim, J.; Yu, Z.; Takahashi, T.; Cho, G.; Javey, A. Fully Printed, High Performance Carbon Nanotube Thin-Film Transistors on Flexible Substrates. Nano Lett. 2013, 13, 3864-3869.

(26) Wang, C.; Hwang, D.; Yu, Z.; Takei, K.; Park, J.; Chen, T.; Ma, B.; Javey, A. User-Interactive Electronic Skin for Instantaneous Pressure Visualization. Nat. Mater. 2013, 12, 899-904.

(27) Kim, S.; Kim, S.; Park, J.; Ju, S.; Mohammadi, S. Fully Transparent Pixel Circuits Driven by Random Network Carbon Nanotube Transistor Circuitry. ACS Nano 2010, 4 (6), 2994-2998.

(28) Ishikawa, F. N.; Curreli, M.; Olson, C. A.; Liao, H.-I.; Sun, R.; Roberts, R. W.; Cote, R. J.; Thompson, M. E.; Zhou, C. Importance of Controlling Nanotube Density for Highly Sensitive and Reliable Biosensors Functional in Physiological Conditions. ACS Nano 2010, 4 (11), 6914-6922.

(29) Collins, P. G.; Bradley, K.; Ishigami, M.; Zettl, A. Extreme Oxygen Sensitivity of Electronic Properties of Carbon Nanotubes. Science 2000, 287, 1801-1804.

(30) Dai, H. Carbon Nanotubes: Opportunities and Challenges. Surf. Sci. 2002, 500, 218-241.

(31) Kim, W.; Javey, A.; Vermesh, O.; Wang, Q.; Li, Y.; Dai, H. Hysteresis Caused by Water Molecules in Carbon Nanotube FieldEffect Transistors. Nano Lett. 2003, 3 (2), 193-198.

(32) Lee, J. S.; Ryu, S.; Yoo, K.; Choi, I. S.; Yun, W. S.; Kim, J. Origin of Gate Hysteresis in Carbon Nanotube Field-Effect Transistors. J. Phys. Chem. C 2007, 111, 12504-12507.

(33) Cho, S.; Lee, K.; Heeger, A. J. Extended Lifetime of Organic Field-Effect Transistors Encapsulated with Titanium Sub-Oxide as an Active Passivation/Barrier layer. Adv. Mater. 2009, 21, 1941-1944.

(34) Kim, S.; Carpenter, P. D.; Jean, R. K.; Chen, H.; Zhou, C.; Ju, S.; Janes, D. B. Role of Self-Assembled Monolayer Passivation in Electrical Transport Properties and Flicker Noise of Nanowire Transistors. ACS Nano 2012, 6 (8), 7352-7361.

(35) Sun, R. C.; Clemens, J. T.; Nelson, J. T. Effects of Silicon Nitride Encapsulation on MOS Device Stability. IEEE Int. Reliab. Phys. Symp. Proc. 1980, 244-251.

(36) Late, D. J.; Liu, B.; Matte, H. S. S. R.; David, V. P.; Rao, C. N. R. Hysteresis in Single-Layer $\mathrm{MoS}_{2}$ Field Effect Transistors. ACS Nano 2012, 6, 5635-5641.

(37) Helbling, T.; Hierold, C.; Roman, C.; Durrer, L.; Mattmann, M.; Beight, V. M. Long Term Investigations of Carbon Nanotube Transistors Encapsulated by Atomic-Layer-Deposited $\mathrm{Al}_{2} \mathrm{O}_{3}$ for Sensor Applications. Nanotechnology 2009, 20, 1-10.

(38) Kim, S. K.; Xuan, Y.; Ye, P. D.; Mohammadi, S.; Back, J. H.; Shim, M. Atomic Layer Deposited $\mathrm{Al}_{2} \mathrm{O}_{3}$ for Gate Dielectric and Passivation Layer of Sinlge-Walled Carbon Nanotube Transistors. Appl. Phys. Lett. 2007, 90, 163108.

(39) Yu, W. J.; Lee, S. Y.; Chae, S. H.; Perello, D.; Han, G. H.; Yun, M.; Lee, Y. H. Small Hysteresis Nanocarbon-Based Integrated Circuits on Flexible and Transparent Plastic Substrate. Nano Lett. 2011, 11, 1344-1350.

(40) Jin, S. H.; Islam, A. E.; Kim, T.-I.; Kim, J.-H.; Alam, M. A.; Rogers, J. A. Sources of Hysteresis in Carbon Nanotube Field-Effect Transistors and Their Elimination via Methylsiloxane Encapsulants and Optimized Growth Procedures. Adv. Funct. Mater. 2012, 22, 2276-2284.

(41) Ong, H. G.; Cheah, J. W.; Zou, X.; Li, B.; Cao, X. H.; Tantang, H.; Li, L.-J.; Zhang, H.; Han, G. C.; Wang, J. Origin of Hysteresis in the Transfer Characteristics of Carbon Nanotube Field-Effect Transistor. J. Phys. D: Appl. Phys. 2011, 44, 285301.

(42) Muoth, M.; Helbling, T.; Durrer, L.; Lee, S.-W.; Roman, C.; Hierold, C. Hysteresis-Free Operation of Suspended Carbon Nanotube Transistors. Nat. Mater. 2010, 5, 589-592.

(43) Shimauchi, H.; Ohno, Y.; Kishimoto, S.; Mizutani, T. Suppression of Hysteresis in Carbon Nanotube Field-Effect Transistors: Effect of Contamination Induced by Device Fabrication Process. Jpn. J. Appl. Phys. 2006, 45, 5501-5503. 
(44) Pascal-Levy, Y.; Shifman, E.; Pal-Chowdhury, M.; Kalifa, I.; Rabkin, T.; Shtempluck, O.; Razin, A.; Kochetkov, V.; Yaish, Y. E. Water-Assisted Mobile Charge Induced Screening and Origin of Hysteresis in Carbon Nanotube Field-Effect Transistors. Phys. Rev. B 2012, 86, 115444.

(45) Mamedov, A. A.; Kotov, N. A.; Prato, M.; Guldi, D. M.; Wicksted, J. P.; Hirsch, A. Molecular Design of Strong Single-Wall Carbon Nanotube/Polyelectrolyte Multilayer Composites. Nat. Mater. 2002, 1, 190-194.

(46) Tsukruk, V. V.; Bliznyuk, V. N. Adhesive and Friction Forces between Chemically Modified Silicon and Silicon Nitride Surfaces. Langmuir 1998, 14 (2), 446-455.

(47) Vosgueritchian, M.; LeMieux, M. C.; Dodge, D.; Bao, Z. Effect of Surface Chemistry on Electronic Properties of Carbon Nanotube Network Thin Film Transistors. ACS Nano 2010, 4, 6137-6145.

(48) Sangwan, V. K.; Ortiz, R. P.; Alaboson, J. M. P.; Emery, J. D.; Bedzyk, M. J.; Lauhon, L. J.; Marks, T. J.; Hersam, M. C. Fundamental Performance Limits of Carbon Nanotube Thin-Film Transistors Achieved Using Hybrid Molecular Dielectrics. ACS Nano 2012, 6, 7480-7488.

(49) Franklin, A. D.; Tulevski, G. S.; Han, S.-J.; Shahrjerdi, D.; Cao, Q.; Chen, H.-Y.; Philip Wong, H.-S.; Haensch, W. Variability in Carbon Nanotube Transistors: Improving Device-to-Device Consistency. ACS Nano 2012, 6, 1109-1115.

(50) Zhang, H.; Weber, S. G. Teflon AF materials. Top. Curr. Chem. 2012, 308, 307-338.

(51) Cheng, X.; Caironi, M.; Noh, Y. Y.; Wang, J.; Newman, C.; Yan, H.; Facchetti, A.; Sirringhaus, H. Air Stable Cross-Linked Cytop Ultrathin Gate Dielectric for High Yield Low-Voltage Top-Gate Organic Field-Effect Transistors. Chem. Mater. 2010, 22, 1559-1566.

(52) Jang, J.; Kim, J. W.; Park, N.; Kim, J.-J. Air Stable C60 based NType Organic Field Effect Transistor Using a Perfluoropolymer Insulator. Org. Electron. 2008, 9, 481-486.

(53) Rolland, A.; Richard, J.; Kleider, J. P.; Mencaraglia, D. Electrical Properties of Amorphous Silicon Transistors and MIS-Devices: Comparative Study of Top Nitride and Bottom Nitride Configurations. J. Electrochem. Soc. 1993, 140, 3679-3683.

(54) Wang, C.; Chien, J.-C.; Takei, K.; Takahashi, T.; Nah, J.; Niknejad, A. M.; Javey, A. Extremely Bendable, High-Performance Integrated Circuits Using Semiconducting Carbon Nanotube Networks for Digital, Analog, and Radio-Frequency Applications. Nano Lett. 2012, 12, 1527-1533.

(55) Veres, J.; Ogier, S. D.; Leeming, S. W.; Cupertino, D. C.; Mohialdin Khaffaf, S. Low-k Insulators as the Choice of Dielectrics in Organic Field-Effect Transistors. Adv. Funct. Mater. 2003, 13, 199204.

(56) Someya, T.; Dodabalapur, A.; Huang, J.; See, K. C.; Katz, H. E. Chemical and Physical Sensing by Organic Field-Effect Transistors and Related Devices. Adv. Mater. 2010, 22, 3799-3811.

(57) Jurchescu, O. D.; Popinciuc, M.; van Wees, B. J.; Palstra, T. T. M. Interface-Controlled, High-Mobility Organic Transistors. Adv. Mater. 2007, 19, 688-692.

(58) Tuan, H. C.; Thompson, M. J.; Johnson, N. M.; Lujan, R. A. Dual-Gate a-Si:H Thin Film Transistors. IEEE Electron Device Lett. 1982, 3 (12), 357-350. 